\title{
Towards an Electromagnetic Crystal Green Function Multiple Scattering Technique for Arbitrary Polarizations, Lattices, and Defects
}

\author{
D. Pissoort $* 1$ and E. Michielssen ${ }^{2}$ \\ ${ }^{1}$ Department of Information Technology, Ghent University, Ghent, Belgium \\ ${ }^{2}$ Department of Electrical Engineering and Computer Science, University of \\ Michigan, Ann Arbor, USA
}

\section{Introduction}

An electromagnetic crystal (EC) Green function multiple scattering technique (MST) that permits the fast simulation of wave propagation in 2D EC devices was presented in [1]. The targeted devices were obtained by removing cylinders from doubly periodic, defect-less, and infinite ECs. Contrary to the conventional free-space Green function MST, which associates unknown currents with the surfaces of all physical cylinders that define the EC device and then subsequently describes their interactions using a free-space Green function, the EC Green function MST considers unknown currents on the surfaces of fictitious, removed cylinders and then models their interaction via a Green function innate to the surrounding infinite EC. For frequencies in the electromagnetic bandgap, this EC Green function decays exponentially with distance. Therefore, its pre-computation can be achieved using the freespace Green function MST by considering a centrally excited, finite, and small EC. For the same reason, the EC Green function MST's system of equations comprises a sparse interaction matrix and a localized excitation that can be solved rapidly by multi-frontal methods. This paper details a generalized formulation of the scheme of [1] that handles EC devices defined on square or triangular lattices supporting both $\mathrm{TM}_{z}$ and $\mathrm{TE}_{z}$ polarized fields, which are obtained by replacing cylinders from a defect-less EC by cylinders that $d o$ not conform to those of the EC background. Throughout the paper it is assumed that the angular frequency $\omega$ lies within the EC bandgap.

\section{Formulation}

Consider a 2D EC device (Fig. 1(a)) obtained by replacing $N_{r}$ cylinders from an infinite and defect-less EC by cylinders that do not conform to those of the EC background; these cylinders henceforth will be termed irregular. To simplify notation, it is assumed that all irregular cylinders are identical. The background EC comprises identical, infinite and $z$-invariant, homogeneous, dielectric/magnetic circular cylinders with radius $r$ and constitutive parameters $\left(\epsilon_{2}, \mu_{2}\right)$ that snap to a regular square or triangular lattice (with lattice constant $a$ ) and reside in a homogeneous background medium with constitutive parameters $\left(\epsilon_{1}, \mu_{1}\right)$. The irregular cylinders' radius is $\tilde{r}$ and their constitutive parameters are $\left(\epsilon_{3}, \mu_{3}\right)$. Define $r_{\max }=\max (r, \tilde{r})$. Let $\boldsymbol{\rho}=(\rho, \phi)$ denote a global position vector in the $x y$-plane. Unit vectors in this global cylindrical coordinate system are denoted by $\boldsymbol{u}_{\rho}$ and $\boldsymbol{u}_{\phi}$. Further, let $\boldsymbol{\rho}_{j}=\left(\rho_{j}, \phi_{j}\right)$ denote a local position vector w.r.t. the center $\boldsymbol{\rho}_{j}^{c}$ of cylinder $j$, $j=1, \ldots, N_{r}$. Unit vectors in this local cylindrical coordinate system are denoted by $\boldsymbol{u}_{\rho_{j}}$ and $\boldsymbol{u}_{\phi_{j}}$. Finally, let $(\epsilon(\boldsymbol{\rho}), \mu(\boldsymbol{\rho}))$ denote the distribution of the constitutive parameters in the $x y$-plane, viz. $(\epsilon(\boldsymbol{\rho}), \mu(\boldsymbol{\rho}))=\left(\epsilon_{1}, \mu_{1}\right)$ outside all physical cylinders, $(\epsilon(\boldsymbol{\rho}), \mu(\boldsymbol{\rho}))=\left(\epsilon_{2}\right.$, $\left.\mu_{2}\right)$ inside a regular cylinder, and $(\epsilon(\boldsymbol{\rho}), \mu(\boldsymbol{\rho}))=\left(\epsilon_{3}, \mu_{3}\right)$ inside an irregular cylinder. 


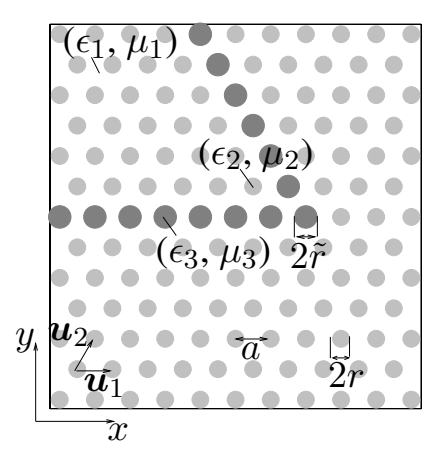

(a) Original configuration

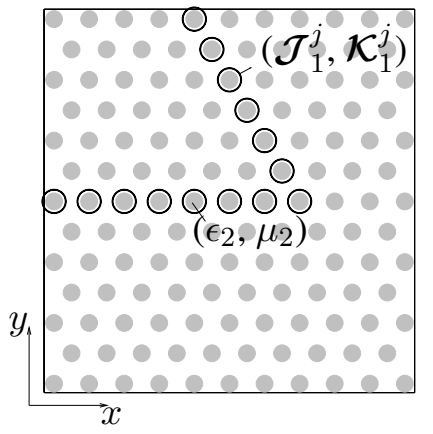

(b) Exterior problem

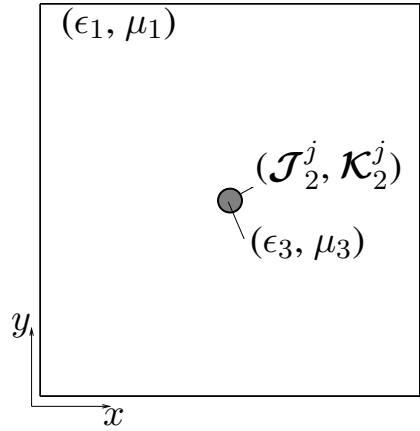

(c) Interior problem

Figure 1: Field-equivalence theorem applied to the simulation of an EC device using the EC Green function approach.

Let $\boldsymbol{E}^{\mathrm{i}}(\boldsymbol{\rho})$ and $\boldsymbol{H}^{\mathrm{i}}(\boldsymbol{\rho})$ denote incident electric and magnetic fields generated by impressed sources that radiate in the presence of the defect-less EC, viz. the infinite EC without any cylinders replaced. Upon replacing the cylinders, total fields $\boldsymbol{E}^{\mathrm{t}}(\boldsymbol{\rho})$ and $\boldsymbol{H}^{\mathrm{t}}(\boldsymbol{\rho})$ are observed. The difference between the total and incident fields defines the scattered fields $\boldsymbol{E}^{\mathrm{s}}(\boldsymbol{\rho})$ and $\boldsymbol{H}^{\mathrm{s}}(\boldsymbol{\rho})$. To describe scattered and total fields, two sets $(\alpha=1,2)$ of equivalent electric and magnetic currents $\left(\mathcal{J}_{\alpha}^{j}(\boldsymbol{\rho}), \mathcal{K}_{\alpha}^{j}(\boldsymbol{\rho})\right)$ are introduced on the circular surface $S_{j}$ with radius $r_{\max }$ round about about every replaced cylinder $j, j=1, \ldots, N_{r}$ (Figs. 1(b) and 1(c)). These currents are related to the total field on $S_{j}$ by

$$
\begin{aligned}
& \mathcal{J}_{\alpha}^{j}(\boldsymbol{\rho})=s_{\alpha} \boldsymbol{u}_{\rho_{j}} \times\left.\boldsymbol{H}^{t}(\boldsymbol{\rho})\right|_{\boldsymbol{\rho} \in S_{j}} \delta\left(\rho_{j}-r_{\max }\right), \\
& \mathcal{K}_{\alpha}^{j}(\boldsymbol{\rho})=-s_{\alpha} \boldsymbol{u}_{\rho_{j}} \times\left.\boldsymbol{E}^{t}(\boldsymbol{\rho})\right|_{\boldsymbol{\rho} \in S_{j}} \delta\left(\rho_{j}-r_{\max }\right),
\end{aligned}
$$

with $s_{1}=1$ and $s_{2}=-1$. The analysis of wave propagation in 2D EC devices falls apart into two non-interacting transverse to $z\left(\mathrm{TM}_{z}\right.$ and $\left.\mathrm{TE}_{z}\right)$ polarizations. However, because of duality, the same formulation can be applied for both polarizations if the Maxwell equations are re-expressed in function of "new" field components $\left(\boldsymbol{F}^{\tau}(\boldsymbol{\rho}), \boldsymbol{G}^{\tau}(\boldsymbol{\rho})\right), \tau=\mathrm{i}, \mathrm{t}, \mathrm{s}$, currents $\left(\mathcal{P}_{\alpha}^{j}(\boldsymbol{\rho}), \mathcal{Q}_{\alpha}^{j}(\boldsymbol{\rho})\right)$, and constitutive parameters $(\kappa(\boldsymbol{\rho}), \theta(\boldsymbol{\rho}))$. For the $\mathrm{TM}_{z}$ polarization, these generalized field components, currents, and constitutive parameters are defined as

$$
\begin{gathered}
F_{z}^{\tau}(\boldsymbol{\rho})=E_{z}^{\tau}(\boldsymbol{\rho}), G_{\rho}^{\tau}(\boldsymbol{\rho})=H_{\rho}^{\tau}(\boldsymbol{\rho}), G_{\phi}^{\tau}(\boldsymbol{\rho})=H_{\phi}^{\tau}, \tau=\mathrm{i}, \mathrm{t}, \mathrm{s} \\
\mathcal{P}_{\alpha, z}^{j}(\boldsymbol{\rho})=\mathcal{J}_{\alpha, z}^{j}(\boldsymbol{\rho}), \mathcal{Q}_{\alpha, \phi_{j}}^{j}(\boldsymbol{\rho})=\mathcal{K}_{\alpha, \phi_{j}}^{j}(\boldsymbol{\rho}), \\
\kappa(\boldsymbol{\rho})=\epsilon(\boldsymbol{\rho}), \theta(\boldsymbol{\rho})=\mu(\boldsymbol{\rho}),
\end{gathered}
$$

while for the $\mathrm{TE}_{z}$ polarization, they are defined as

$$
\begin{gathered}
F_{z}^{\tau}(\boldsymbol{\rho})=-H_{z}^{\tau}(\boldsymbol{\rho}), G_{\rho}^{\tau}(\boldsymbol{\rho})=E_{\rho}^{\tau}(\boldsymbol{\rho}), G_{\phi}^{\tau}(\boldsymbol{\rho})=E_{\phi}^{\tau}(\boldsymbol{\rho}), \tau=\mathrm{i}, \mathrm{t}, \mathrm{s} \\
\mathcal{P}_{\alpha, z}^{j}(\boldsymbol{\rho})=-\mathcal{K}_{\alpha, z}^{j}(\boldsymbol{\rho}), \mathcal{Q}_{\alpha, \phi_{j}}^{j}(\boldsymbol{\rho})=\mathcal{J}_{\alpha, \phi_{j}}^{j}(\boldsymbol{\rho}), \\
\kappa(\boldsymbol{\rho})=\mu(\boldsymbol{\rho}), \theta(\boldsymbol{\rho})=\epsilon(\boldsymbol{\rho}) .
\end{gathered}
$$

All other field components are zero. Moreover, the formulation is essentially the same for square and triangular lattices if the latter is described in function of the so-called lattice unit vectors $\boldsymbol{u}_{1}$ and $\boldsymbol{u}_{2}$ (Fig. 1(a)). 
If $F_{\alpha, z}^{\mathrm{s}, j}(\boldsymbol{\rho})$ denotes the amplitude of the $z$-directed field radiated jointly by $\mathcal{P}_{\alpha, z}^{j}(\boldsymbol{\rho})$ and $\mathcal{Q}_{\alpha, \phi_{j}}^{j}(\boldsymbol{\rho})$ in the unbounded and defect-less EC when $\alpha=1$ or radiated by $\mathcal{P}_{\alpha, z}^{j}(\boldsymbol{\rho})$ and $\mathcal{Q}_{\alpha, \phi_{j}}^{j}(\rho)$ around an irregular cylinder placed in the unbounded medium with constitutive parameters $\left(\kappa_{1}, \theta_{1}\right)$ when $\alpha=2$, then it follows from the field-equivalence theorem that

$$
\begin{aligned}
F_{z}^{\mathrm{i}}(\boldsymbol{\rho})+\sum_{j=1}^{N_{r}} F_{1, z}^{\mathrm{s}, j}(\boldsymbol{\rho}) & =0 \text { if } \boldsymbol{\rho} \in S_{i}^{-}, i=1, \ldots, N_{r}, \\
F_{2, z}^{\mathrm{s}, j}(\boldsymbol{\rho}) & =0 \text { if } \boldsymbol{\rho} \in S_{j}^{+}, j=1, \ldots, N_{r} .
\end{aligned}
$$

Here, $S_{j}^{-}$and $S_{j}^{+}$denote surfaces residing just inside and outside the surface $S_{j}$, respectively. To solve equations (9-10), $\mathcal{P}_{\alpha, z}^{j}(\boldsymbol{\rho})$ and $\mathcal{Q}_{\alpha, \phi_{j}}^{j}(\boldsymbol{\rho})$ are expanded as

$$
\begin{aligned}
\mathcal{P}_{\alpha, z}^{j}(\boldsymbol{\rho}) & =s_{\alpha} \sum_{n=-K}^{K} \frac{P_{n}^{j}}{2 \pi r_{\max }} e^{j n \phi_{j}} \delta\left(\rho_{j}-r_{\max }\right), \\
\mathcal{Q}_{\alpha, \phi_{j}}^{j}(\boldsymbol{\rho}) & =s_{\alpha} \sum_{n=-K}^{K} \frac{Q_{n}^{j}}{2 \pi r_{\max }} e^{j n \phi_{j}} \delta\left(\rho_{j}-r_{\max }\right) .
\end{aligned}
$$

Solving the (interior) eqn. (10) yields

$$
\frac{P_{n}^{j}}{Q_{n}^{j}}=-j \sqrt{\frac{\kappa_{1}}{\theta_{1}}} \frac{\mathcal{N}_{13}^{n}(\tilde{r}) J_{n}^{\prime}\left(k_{1} r_{\max }\right)-\mathcal{T}_{13}^{n}(\tilde{r}) H_{n}^{(2)^{\prime}}\left(k_{1} r_{\max }\right)}{\mathcal{N}_{13}^{n}(\tilde{r}) J_{n}\left(k_{1} r_{\max }\right)-\mathcal{T}_{13}^{n}(\tilde{r}) H_{n}^{(2)}\left(k_{1} r_{\max }\right)} .
$$

Here,

$$
\begin{aligned}
\mathcal{N}_{13}^{n}(\tilde{r}) & =\kappa_{3} \theta_{1} H_{n}^{(2)}\left(k_{1} \tilde{r}\right) J_{n}^{\prime}\left(k_{3} \tilde{r}\right)-\kappa_{1} \theta_{3} H_{n}^{(2)^{\prime}}\left(k_{1} \tilde{r}\right) J_{n}\left(k_{3} \tilde{r}\right) \\
\mathcal{T}_{13}^{n}(\tilde{r}) & =\kappa_{3} \theta_{1} J_{n}\left(k_{1} \tilde{r}\right) J_{n}^{\prime}\left(k_{3} \tilde{r}\right)-\kappa_{1} \theta_{3} J_{n}^{\prime}\left(k_{1} \tilde{r}\right) J_{n}\left(k_{3} \tilde{r}\right)
\end{aligned}
$$

Relationship (13) permits $F_{1, z}^{\mathrm{s}, j}(\boldsymbol{\rho})$, the amplitude of the $z$-directed field radiated jointly by $\mathcal{P}_{1, z}^{j}(\boldsymbol{\rho})$ and $\mathcal{Q}_{1, \phi_{j}}^{j}(\boldsymbol{\rho})$ in the defect-less and unbounded $E C$, to be expressed as

$$
F_{1, z}^{\mathrm{s}, j}(\boldsymbol{\rho})=\sum_{n=-K}^{K} G_{n}^{\mathrm{ec}}\left(\boldsymbol{\rho}_{j}\right) P_{n}^{j} .
$$

Quantity $G_{n}^{\mathrm{ec}}(\boldsymbol{\rho})$ is the EC Green function for a Huygens source with distributed components $\frac{1}{2 \pi r_{\max }} \delta\left(\rho-r_{\max }\right) e^{j n \phi} \boldsymbol{u}_{z}$ and $\frac{j}{2 \pi r_{\max }} \sqrt{\frac{\theta_{1}}{\kappa_{1}}}\left[\frac{\mathcal{N}_{13}^{n}(\tilde{r}) J_{n}\left(k_{1} r_{\max }\right)-\mathcal{T}_{13}^{n}(\tilde{r}) H_{n}^{(2)}\left(k_{1} r_{\max }\right)}{\mathcal{N}_{13}^{n}(\tilde{r}) J_{n}^{\prime}\left(k_{1} r_{\max }\right)-\mathcal{T}_{13}^{n}(\tilde{r}) H_{n}^{(2)}\left(k_{1} r_{\max }\right)}\right] \delta(\rho-$ $\left.r_{\max }\right) e^{j n \phi} \boldsymbol{u}_{\phi}$ radiating jointly in the defect-less and unbounded EC. To solve for the unknowns $P_{n}^{j}$, expansion (16) is inserted into (9) and the resulting equation is tested by $T_{m}^{i}(\boldsymbol{\rho})=\frac{1}{2 \pi J_{m}\left(k_{2} r_{\max }\right)} e^{-j m \phi_{i}} \delta\left(\rho_{i}-r_{\max }\right)$, resulting in the matrix equation

$$
Z P=F,
$$

where $Z_{m n}^{i j}=\left\langle T_{m}^{i}(\boldsymbol{\rho}), G_{n}^{\mathrm{ec}}\left(\boldsymbol{\rho}_{j}\right)\right\rangle$ and $F_{m}^{i}=\left\langle T_{m}^{i}(\boldsymbol{\rho}),-F_{z}^{\mathrm{i}}(\boldsymbol{\rho})\right\rangle$. The interaction matrix $\boldsymbol{Z}$ is essentially sparse. Because $\omega$ is assumed to lie within the EC bandgap, $G_{n}^{\text {ec }}(\boldsymbol{\rho})$ decays exponentially with $|\boldsymbol{\rho}|$. Therefore, each and every removed cylinder only interacts with 
its near neighbors, thereby rendering vanishingly small all entries of $\boldsymbol{Z}$ describing interactions between sufficiently separated replaced cylinders. Recently, significant advances in direct methods for inverting such sparse matrices have been reported, e.g., the multi-frontal method by Duff and Reid [2]. Unfortunately, no closed-form expressions for $G_{n}^{\mathrm{ec}}(\boldsymbol{\rho})$ exist. As already mentioned above, $G_{n}^{\mathrm{ec}}(\boldsymbol{\rho})$ decays exponentially with $|\boldsymbol{\rho}|$. As a result, $G_{n}^{\mathrm{ec}}(\boldsymbol{\rho})$ can be evaluated using the conventional free-space MST by considering a finite EC wherein the central cylinder, which is assumed centered about the spatial origin $\boldsymbol{\rho}=\mathbf{0}$, is excited by the above defined Huygens source. As this excitation depends on the radius and constitutive parameters of the irregular cylinder, this means that for EC devices comprising different types of irregular cylinders many different EC Green functions have to be calculated. Fortunately, all these EC Green functions can be computed quickly starting from the EC Green function for a removed cylinder (as defined in [1]) using FFTs, making it unnecessary to solve a new linear system for every type of EC Green function.

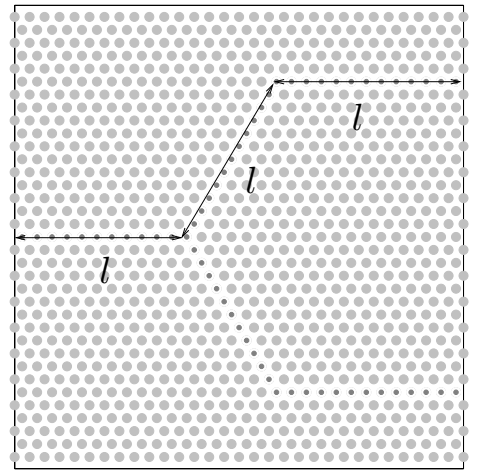

(a) Configuration

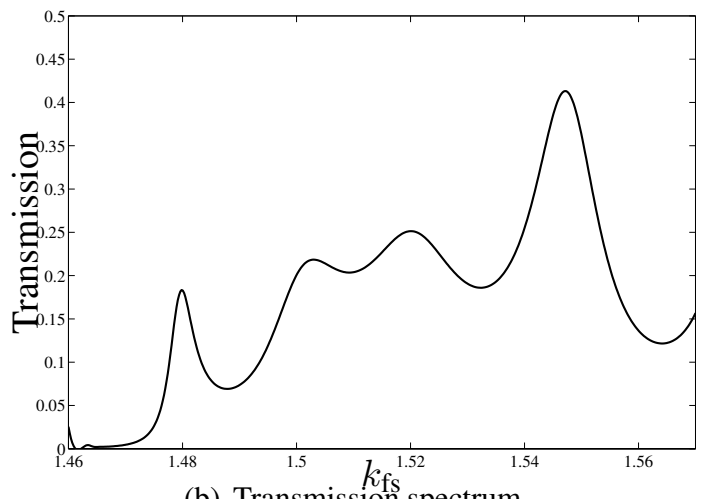

(b) Transmission spectrum

Figure 2: Transmission spectrum of an EC waveguide splitter calculated with the EC Green function MST.

\section{Example}

To demonstrate the usefulness of the proposed scheme, consider the EC waveguide splitter depicted in Fig. 2(a). The background EC is composed of air holes, i.e., $\left(\epsilon_{2}, \mu_{2}\right)=\left(\epsilon_{0}\right.$, $\left.\mu_{0}\right)$, with radius $r=0.35 a$ that are arranged on a triangular lattice with lattice constant $a$ and reside in a background medium with $\left(\epsilon_{1}, \mu_{1}\right)=\left(11.56 \epsilon_{0}, \mu_{0}\right)$. The EC waveguides, with length $l=20 a$, comprise smaller air holes with radius $\tilde{r}=0.07 a$. Figure 2(b) shows the transmission spectrum for $\mathrm{TE}_{z}$ polarized fields of the $\mathrm{EC}$ waveguide splitter calculated with the EC Green function MST. With $K=2$, the EC Green function MST calls for 500 unknowns. For one frequency point, it takes roughly $30 \mathrm{~s}$ to calculate the EC Green function and $1 \mathrm{~s}$ to fill and solve the sparse system of equations (17).

\section{References}

[1] D. Pissoort, E. Michielssen, F. Olyslager, and D. De Zutter, "Fast analysis of 2D electromagnetic crystal structures using a periodic Green function approach," IEEE J. Lightwave Technol., vol. 23, pp. 2294-2308, July 2005.

[2] I. Duff and J. Reid, "The multifrontal solution of unsymmetric sets of linear equations," SIAM J. Sci. Comput., vol. 5, pp. 633-641, 1984. 


\section{IEEE Antennas and Propagation Society International Symposium 2006}
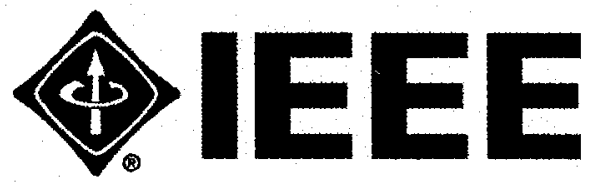

Copyright and Reprint Permissions: Abstracting is permitted with credit to the source. Libraries are permitted to photocopy beyond the limit of U.S. copyright law for private use of patrons those articles in this volume that carry a code at the bottom of the first page, provided the per-copy fee indicated in thecode is paid through Copyright Clearance Center, 222 Rosewood Drive, Danvers, MA 01923.

For other copying, reprint or republications permission, write to IEEE Copyrights Manager, IEEE Operations Center, 445 Hoes Lane, Piscataway, New Jersey USA 08854. All rights reserved.

IEEE Catalog Number:

ISBN:

Library of Congress No.:
$06 \mathrm{CH} 37758 \mathrm{C}$

1-4244-0123-2

$90-640397$ 
2006 IEEE Antennas and Propagation Society International Symposium

\title{
with \\ USNC/URSI National Radio Science and AMEREM Meetings
}

\section{Symposium Digest Volume 3 of 5}

\author{
9-14 July 2006
} Albuquerque Convention Center

\author{
and \\ Hyatt Regency Hotel
Albuquerque, NM USA \\ Hyatt Regency Hotel
Albuquerque, NM USA
}

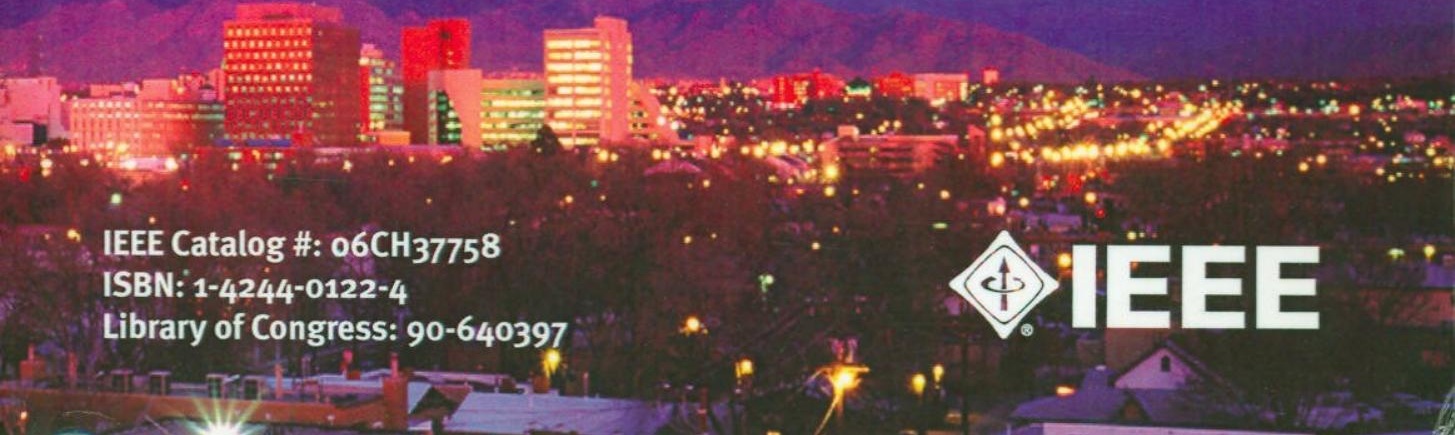

